\title{
Neutral Hydrogen in the Magellanic Clouds
}

\author{
L. Staveley-Smith
}

Australia Telescope National Facility, CSIRO, P.O. Box 76, Epping, NSW 1710, Australia

\section{S. Kim}

\author{
Astronomy Department, University of Illinois at Urbana-Champaign, \\ Urbana, IL 61801, USA
}

\section{S. Stanimirović}

University of Western Sydney Nepean, P.O. Box 10, Kingswood, NSW 2747, Australia

\begin{abstract}
We review observations of neutral atomic hydrogen (HI) in the Magellanic Clouds (MCs). Being the nearest gas-rich neighbours of the Milky Way the MCs give us an excellent opportunity to study in detail the structure and evolution of the interstellar medium (ISM) and the effect of interactions between galaxies. $\mathrm{HI}$ in emission provides a probe of the structure and velocity field of the Clouds, allowing the study of their velocity dispersion, 3-D structure, and large-scale total-mass distribution. Recent data from Australia Telescope Compact Array surveys reveal a morphology (for both Clouds) which is heavily dominated by the effects of local star-formation, rotational shear, fragmentation, self-gravity and turbulence. The new data, which has a spatial resolution down to 10 $\mathrm{pc}$, also allows the study of the distribution functions in velocity and mass for HI clouds. We discuss the morphology, dynamics and giant shell population of the LMC and SMC.
\end{abstract}

\section{Introduction}

In this paper, we concentrate on recent observations of $\mathrm{HI}$ in emission in the LMC and SMC. HI is an extremely important constituent of the ISM in both Magellanic Clouds. Emission in the $\lambda 21 \mathrm{~cm}$ line is independent of temperature for typical conditions and, in the usual optically-thin regime, becomes a direct measure of column density (e.g. Kulkarni \& Heiles 1988). The HI content of the Clouds is high - the HI mass-to-light ratios are 0.14 and $0.61 \mathrm{M}_{\odot} / \mathrm{L}_{\odot}$ for the LMC and SMC, respectively (Staveley-Smith et al. 1998). 


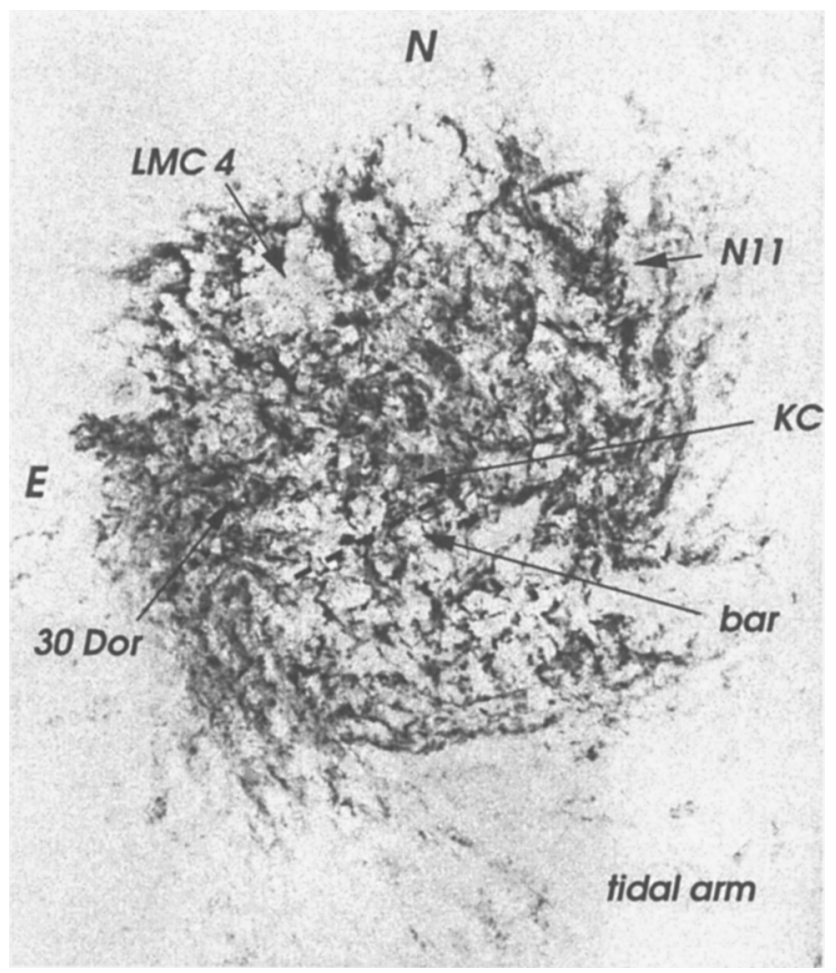

Figure 1. The peak-brightness-temperature image of the LMC from Kim et al. (1998). The HII regions N11 and 30 Doradus are marked, as are the positions of the optical bar and the kinematic center (KC). The tidal arm in the south stretches into the Bridge region between the LMC and SMC. This sub-image is $9.4 \times 10^{\circ} .7$ across.

For the LMC, the highest resolution study is the Australia Telescope Compact Array $^{1}$ (ATCA) mosaic by Kim et al. (1998). This provides an angular resolution of 1.0 and a velocity resolution of $1.6 \mathrm{~km} \mathrm{~s}^{-1}$ over the whole $10^{\circ} \times 12^{\circ}$ region encompassing the LMC. However, this study does not include large-scale features which can only be observed with a single-dish. For this, the observations of Luks \& Rohlfs (1992) and Putman et al. (1999) with the Parkes telescope may be used. In a square-degree region around 30 Dor, Mebold et al. (1997) have obtained high-resolution (30") ATCA data which they have combined with recent Parkes observations. For the SMC, Stanimirović et al. (1999) have combined recent Parkes observations with the ATCA mosaic of Staveley-Smith et al. (1997). This provides full spatial sampling with an angular resolution of 1!6 


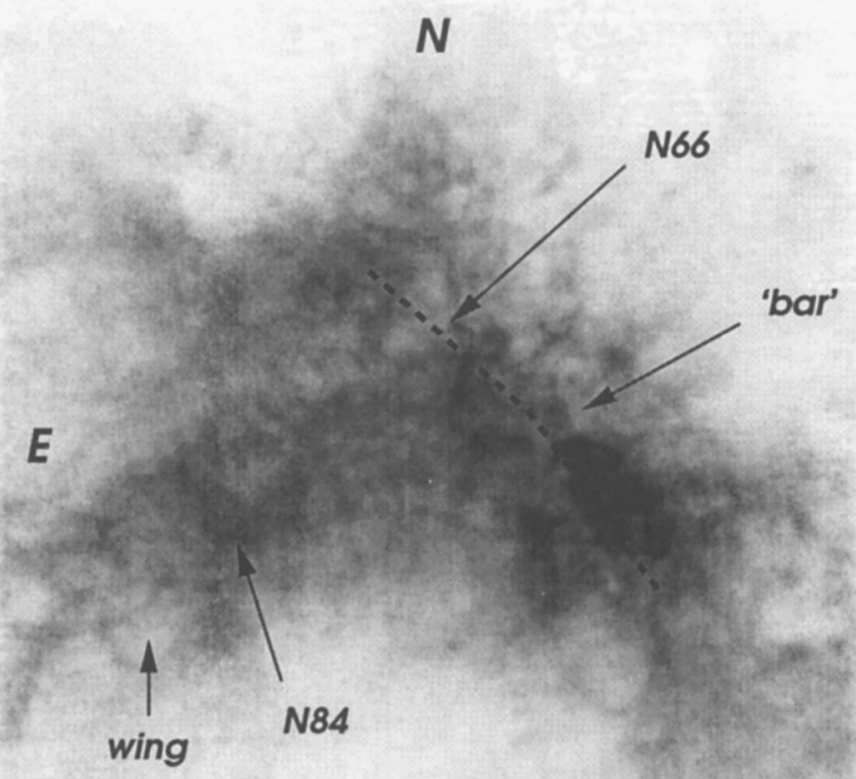

Figure 2. The column density image of the SMC from Stanimirovic et al. (1999). The HII regions N66 and N84 are marked, as well as the positions of the optical bar and the eastern wing. The image is $4.5 \times 4.5$ across.

and a velocity resolution of $1.6 \mathrm{~km} \mathrm{~s}^{-1}$ over a region $4.5 \times 4.5$ encompassing the SMC.

\section{HI Morphology and Dynamics}

The distribution of HI in the LMC is shown in Figure 1. As noted by Kim et al. (1998), the galaxy is surprisingly symmetric and disk-like on the largest scales, compared with earlier optical studies. The outer, tenuous arms reveal its clockwise rotation. The well-known optical bar is not prominent in HI. However, a tidal arm to the south of the LMC is prominent, and appears to extend into the Bridge region connecting the LMC and SMC. The SMC, on the other hand, shows no large-scale symmetry (Figure 2), but is instead dominated by a central HI concentration associated with the optical 'bar', and an eastern wing

\footnotetext{
${ }^{1}$ The Australia Telescope is funded by the Commonwealth of Australia for operation as a National Facility managed by CSIRO.
} 


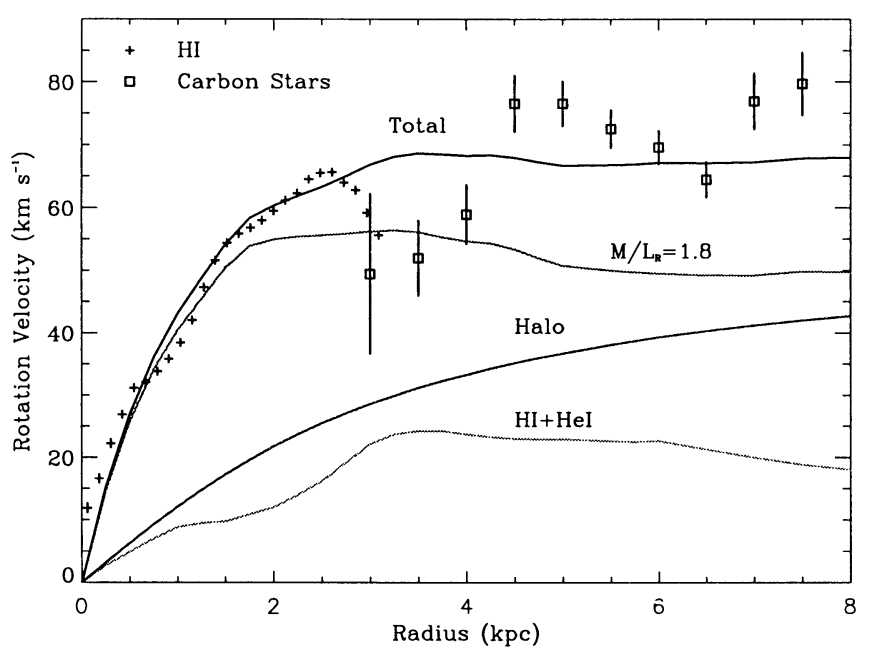

Figure 3. Rotation curve for the LMC from HI (+; Kim et al. 1998) and Carbon star ( $\square$; Kunkel et al. 1997) data. The model rotation curve (topmost solid line) is derived from a thick (scale height $600 \mathrm{pc}$, inclination $33^{\circ}$ ) stellar and $\mathrm{HI} / \mathrm{He}$ disk, and a spherical isothermal halo.

(Stanimirović et al. 1999). On small scales, the ISM of both the LMC and SMC is dominated by the effects of stellar winds and SN, this being clearer in Figure 1 owing to the missing short spacings.

As Figure 3 shows, the rotation curve of the LMC can be decomposed into several significant components: a thick stellar disk, a thick gaseous disk and a dark halo. Within a radius of $4 \mathrm{kpc}$, Figure 4 shows that the respective masses are $1.4 \times 10^{9}, 4 \times 10^{8}$ and $1.0 \times 10^{9} \mathrm{M}_{\odot}$.

The SMC also has a significant velocity gradient (Figure 5). If interpreted as simple, disk-like rotation, the inclination of the SMC would have to be $<35^{\circ}$ in order for the dynamical mass to exceed the obvious baryonic mass (HI, He, stars) of $\sim 1.3 \times 10^{9} \mathrm{M}_{\odot}$ within a $2 \mathrm{kpc}$ radius. This would imply intrinsic rotation $>50 \mathrm{~km} \mathrm{~s}^{-1}$. However it is unlikely that simple disk rotation model is relevant. Cepheid data (Caldwell \& Coulson 1986) show that the average distance of the stellar population in the NE may be closer by $\sim 16 \mathrm{kpc}$ compared with the SW. This, combined with modeling (Gardiner et al. 1994), shows that larger scale tidal forces are likely to be responsible for the current kinematics of the SMC.

\section{The HI Shell Population}

Recent ( $<50 \mathrm{Myr}$ ) star-formation events leave indelible features on the ISM through the effects of stellar radiation, winds and supernova blast waves. In the HI data, these features reveal themselves most clearly as giant and supergiant shells. The physical properties of these features (mass, expansion velocity, radius, dynamical age, kinetic energy, number density, size distribution) can be measured and tested against models of star formation. The size distribution 


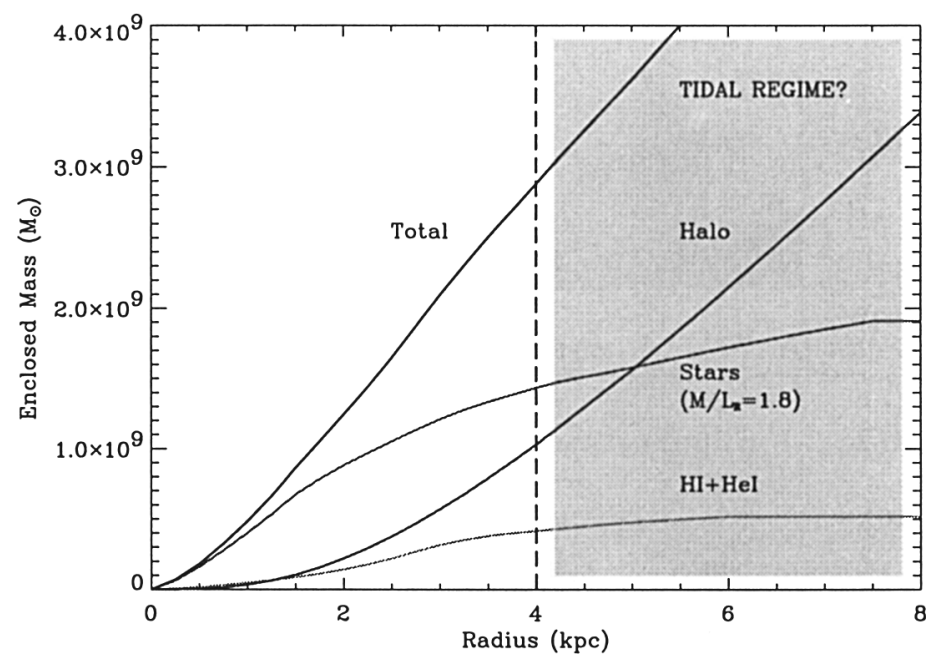

Figure 4. The enclosed masses of stars, gas and halo in the LMC. At $4 \mathrm{kpc}$ radius, the enclosed mass is $3 \times 10^{9} \mathrm{M}_{\odot}$. Beyond $4 \mathrm{kpc}$, it is likely that tidal forces are important.

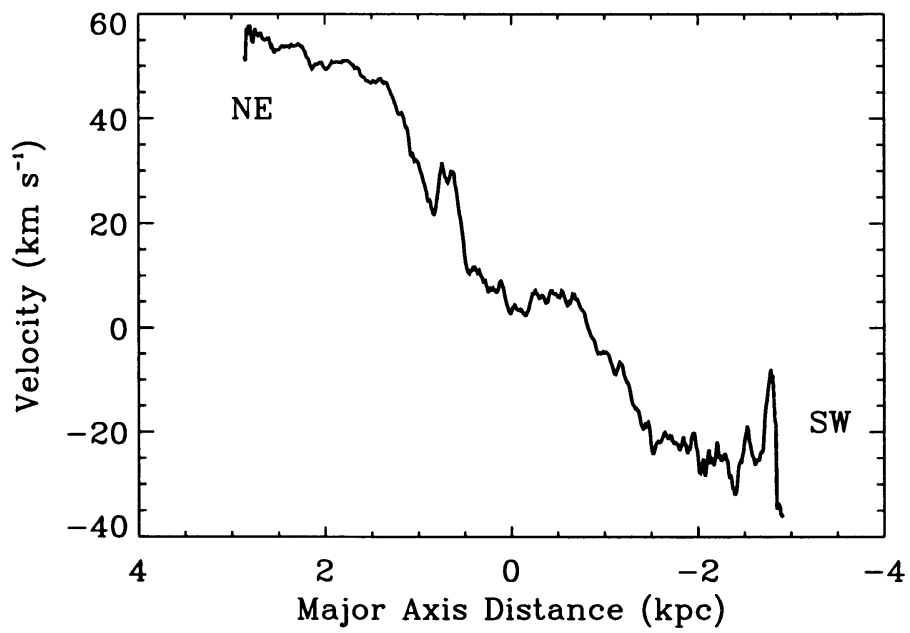

Figure 5. Radial velocities along the kinematic major axis of the SMC. Velocities have been corrected for Proper Motion and are in the Magellanocentric frame of Gardiner et al. (1994).

of the recently cataloged shells and candidate shells in the LMC and SMC is shown in Figure 6. For $R>80 \mathrm{pc}$, a limit dictated by resolution and completeness, the SMC has a shell-size distribution that can be approximated by a power law $N(\log R) \propto R^{-1.6}$. Using the standard model for adiabatically expanding shells (e.g., Weaver et al. 1977), Oey \& Clarke (1997) predict a power law $N(\log R) \propto R^{-1.7 \pm 0.6}$, in good agreement. For the LMC, the size distribution 

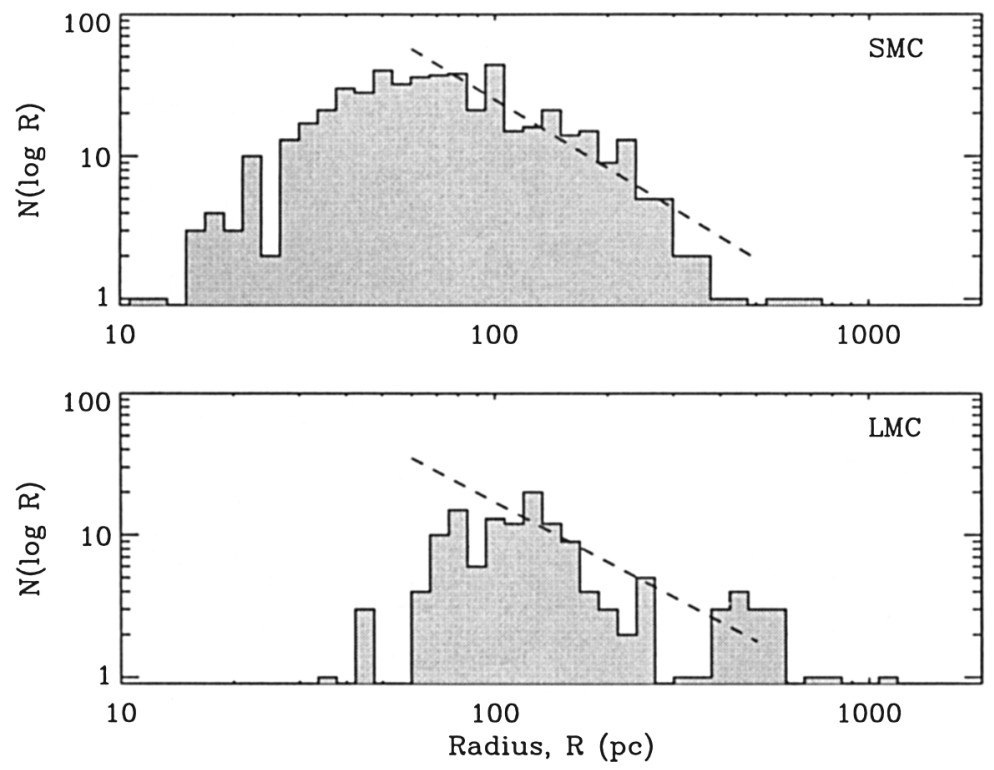

Figure 6. The distribution of shell radii for HI shells in the SMC (top) and HI shell candidates in the LMC (bottom). The dashed line represents a power law $N(\log R) \propto R^{-\alpha}$ with $\alpha=1.6$ for the SMC and $\alpha=1.4$ for the LMC.

for the supergiant and giant shell candidates (Kim et al. 1999) is also shown in Figure 6. There is an apparent excess of supergiant shells in the LMC, resulting in a shallower power law, $N(\log R) \propto R^{-1.4}$. However, the number of SMC shells $(\sim 500$ cataloged $)$ appears to greatly exceed the number of LMC shells $(\sim 130$ : Kim 1998, Kim et al. 1999). At a shell radius of $100 \mathrm{pc}$, the surface density of SMC shells is about 4.1 times that of LMC shells. This is surprising given that the specific star-formation rate of the LMC is probably higher than for the SMC and that, for a given age, shell radius is only a weak function of wind luminosity and ISM density. The surface density ratio of shells is comparable to the ratio of HI surface densities ( 3.8, Staveley-Smith et al. 1998), implying that confusion and a more complex ISM may impede shell identification in the LMC. Another factor may be venting of LMC shells once their radius approaches the scale height of the disk. This causes more shells to stall, and become difficult to identify. Almost half the supergiant shell candidates noted by Kim et al. (1999) have uncertain expansion velocities, and may be stalled.

Acknowledgments. We thank J. Dickey, M. Dopita, K. Freeman, D. Hatzidimitriou, M. Kesteven, D. McConnell, R. Sault and S. Snowden who have greatly assisted us in this research.

\section{References}

Caldwell, J. A. R., \& Coulson, I. M. 1986, MNRAS, 218, 223 
Gardiner, L. T., Sawa, T., \& Fujimoto, M. 1994, MNRAS, 266, 567

Kim, S. 1998, Ph.D. Thesis, Australian National University

Kim, S., Dopita, M. A., Staveley-Smith, L., \& Bessell, M. 1999, ApJ, submitted Kim, S., Staveley-Smith, L., Dopita, M. A., Freeman, K. C., Sault, R. J., Kesteven, M. J., \& McConnell, D. 1998, ApJ, 503, 674

Kulkarni, S. R., \& Heiles, C. 1988, in Galactic and Extragalactic Radio Astronomy, G. L. Verschuur \& K. I. Kellermann, New York: Springer-Verlag, 95

Kunkel, W. E., Demers, S., Irwin, M. J., \& Albert, L. 1997, ApJ, 488, L129

Luks, T., \& Rohlfs, K. 1992, A\&A, 263, 41

Mebold, U., Düsterberg, C., Dickey, J. M., Staveley-Smith, L., \& Kalberla, P. 1997, ApJ, 490, L65

Oey, M. S., \& Clarke, C. J. 1997, MNRAS, 289, 570

Putman, M. E., Gibson, B. K., \& Staveley-Smith, L. 1999, this volume

Stanimirović, S., Staveley-Smith, L., Dickey, J. M., Sault, R. J., \& Snowden, S. L. 1999, MNRAS, 302, 417

Staveley-Smith, L., Sault, R .J., Hatzidimitriou, D., Kesteven, M. J., \& McConnell, D. 1997, MNRAS, 289, 225

Staveley-Smith, L., Kim, S., Putman, M., \& Stanimirović, S. 1998, in Reviews in Modern Astronomy, R. E. Schielicke, Hamburg: Astronomische Gesellschaft, 117

Weaver, R., McCray, R., Castor, J., Shapiro, P., \& Moore, R. 1977, ApJ, 218, 377

\section{Discussion}

Marc Kutner: When you do mosaicing how do you assure image fidelity on intermediate scales (i.e. structures that extend over a few fields)?

Stavely-Smith: The largest scale structures $\left(00^{\circ}\right)$ are not present in the LMC data presented. On intermedidate scales $(0.3-0.6)$ fidelity is assured by Nyquist sampling of the field (primary beams spaced by half a FWHP beam). A maximum entropy procedure is used to combine information from neighboring fields.

Sally Oey: The relative number of HI shells compared to HII regions in the SMC is consistent with the relative life expectancies for shells vs. HII regions. However, the small number of shells in the LMC is startling, considering the higher star formation rate in the LMC. Why is there such a small number of shells in the LMC?

Stavely-Smith: I suspect the answer to this very interesting question is related to (A) the LMC having a thinner disk than the SMC which allows the easier venting of giant/supergiant shells; and (B) the LMC being much less HI-rich than the SMC. This may decrease the time required for the shells to dissipate into the general ISM.

David Graff: What are the large scale departures from the disk rotation solution in the LMC? 
Stavely-Smith: The most significant departues are around the supergiant shells such as LMC4 and the 30 Dor regions. In the east, there is a spiral arm which is dynamically hot, perhaps a result of tidal interactives.

Daniel Wang: Any idea about the volume filling factor of HI gas?

Stavely-Smith: If we assume the HI shell interiors contain no HI, the volume filling factor of the HI gas is 0.5 , or less in the SMC. If modelled as a fractal structure, a much smaller filling factor is suggested.

Nicholas Suntzeff: Stars in the LMC extend to $\sim 8 \mathrm{kpc}$, while your maps and total $\mathrm{HI}$ gas extend only out to $4 \mathrm{kpc}$. Do you have total HI masses out to the extent of the stellar disk?

Staveley-Smith: The existing ATCA maps have no zero-spacing (single-dish) information, so I cannot say. The previous data of McGee and Milborn and Luks and Rohlfs appear to suggest that the HI mass beyond $4 \mathrm{kpc}$ does not contribute much to the total HI mass. However, there appear to be calibration differences between their data sets. 